\title{
Fixed-point theorems for the sum of two operators under $\omega$-condensing
}

Fuli Wang*

\section{"Correspondence:} win-fully@163.com

School of Mathematics and Physics, Changzhou University, Changzhou, 213164, China

\section{第 Springer}

\begin{abstract}
The purpose of this paper is to establish fixed-point theorems for the sum of two operators $A$ and $B$, where the operator $A$ is assumed to be contractive with respect to the measure of weak noncompactness, while $B$ is an $\varphi$-nonlinear contraction. In the last section, we apply such results to study the existence of solutions to a nonlinear Hammerstein integral equation in $L^{1}$ space.
\end{abstract}

Keywords: measure of weak noncompactness; weak condensing; $\varphi$-nonlinear contraction; nonlinear Hammerstein integral equations

\section{Introduction}

The existence of fixed points for the sum of two operators has been followed with interest for a long time. In 1958, to study the existence of solutions of nonlinear equations of the form

$$
A x+B x=x, \quad x \in M,
$$

Krasnosel'skii [1] first proved operator $A+B$ has a fixed point whenever $M$ is a nonempty closed convex subset of Banach space $X$ and the operators $A$ and $B$ satisfy:

(i) $A$ is continuous on $M$, and $A(M)$ is relatively compact,

(ii) $B$ is a $k$-contraction with $k \in[0,1)$,

(iii) $A(M)+B(M) \subset M$.

In 1955, Darbo [2] extended the Schauder fixed-point theorem to the setting of noncompact operators, introducing the notion of $k$-set contraction. It is not hard to see that the Krasnosel'skii theorem is a particular case of the Darbo theorem. Namely, it appears that $A+B$ is a $k$-set contraction with respect to the Kuratowskii measure of noncompactness. In 1967, Sadovskii [3] gave a fixed-point result more general than the Darbo theorem using the concept of condensing operator.

In 1977, De Blasi [4] introduced the concept of measure of weak noncompactness. Emmanuele [5] established a Sadovskii-type fixed-point result using the concept of $\omega$-condensing with respect to the measure of weak noncompactness, in which the weak continuity of the operator is required. Recently, Garcia-Falset and Latrach established a new version of Sadovskii-type fixed-point theorem for the weakly sequentially continuous operators (see [6, Lemma 3.2]).

On the other hand, since the weak continuity condition is usually not easy to verify, Latrach et al. [7, 8] established generalizations of the Schauder, Darbo and Krasnosel'skii

(c) 2013 Wang: licensee Springer. This is an Open Access article distributed under the terms of the Creative Commons Attribution License (http://creativecommons.org/licenses/by/2.0), which permits unrestricted use, distribution, and reproduction in any medium, provided the original work is properly cited. 
fixed-point theorems for the weak topology. Their analysis uses the concept of the Blasi measure of weak noncompactness. Moreover, and in contrast to previous works, to prove the new versions of the fixed-point theorems, they neither assume the weak continuity nor the weakly sequential continuity of the operators.

The purpose of this paper is to establish several fixed-point theorems for the sum of two operators under $\omega$-condensing. By relaxing the condition of weak compactness of operators, these results extend and supplement some previous ones in the literatures.

This paper is organized as follows. In Section 2, we gather some notions and preliminary facts which will be needed in our further considerations. In Section 3, on the basis of a Sadovskii-type fixed-point theorem for $\omega$-condensing operators and its variant on whole space, we discuss several fixed-point theorems for the sum of $A+B$, where $A$ is a $\omega$-contraction and $B$ is an $\varphi$-nonlinear contraction. In Section 4 , we apply such results to study the existence of solutions to a nonlinear Hammerstein integral equation in $L^{1}$ space.

\section{Preliminaries}

We first gather together some notations and preliminary facts of some weak topology feature which will be needed in our further considerations. Let $\mathcal{B}(X)$ be the collection of all nonempty bounded subsets of a Banach space $X$, and let $\mathcal{W}(X)$ be the subset of $\mathcal{B}(X)$ consisting of all weakly compact subsets of $X$. Also, let $\mathbf{B}_{r}$ denote the closed ball in $X$ centered in $\mathbf{0}$ and with radius $r$.

De Blasi [4] introduced the map $\omega: \mathcal{B}(X) \rightarrow \mathbb{R}^{+}$defined by

$$
\omega\{M\}=\inf \left\{r>0: \exists W \in \mathcal{W}(X): M \subset W+\mathbf{B}_{r}\right\} \quad \text { for } M \in \mathcal{B}(X) .
$$

Before we launch into the details, we recall some important properties needed hereafter for the sake of completeness (for the proofs, we refer the reader to [4] and [9]).

Lemma 2.1 Let $M, M_{1}$ and $M_{2}$ be in $\mathcal{B}(X)$; we have:

(a) $\omega\left\{M_{1}\right\} \leq \omega\left\{M_{2}\right\}$ whenever $M_{1} \subset M_{2}$.

(b) $\omega\{M\}=0$ if and only if $\bar{M}^{w} \in \mathcal{W}(X)$, where $\bar{M}^{w}$ is the weak closure of $M$.

(c) $\omega\left\{\bar{M}^{w}\right\}=\omega\{M\}$.

(d) $\omega\{\operatorname{co}(M)\}=\omega\{M\}$ where $\operatorname{co}(M)$ refers to the convex hull of $M$.

(e) $\omega\{\lambda M\}=|\lambda| \omega\{M\}$, for all $\lambda \in \mathbb{R}$.

(f) $\omega\left\{M_{1}+M_{2}\right\} \leq \omega\left\{M_{1}\right\}+\omega\left\{M_{2}\right\}$.

(g) $\omega\left\{M_{1} \cup M_{2}\right\}=\max \left\{\omega\left\{M_{1}\right\}, \omega\left\{M_{2}\right\}\right\}$.

(h) If $\left(M_{n}\right)_{n=1}^{\infty}$ is a decreasing sequence of nonempty, bounded and weakly closed subsets of $X$ with $\lim _{n \rightarrow \infty} \omega\left\{M_{n}\right\}=0$, then $\lim _{n \rightarrow \infty} \bigcap_{k=1}^{\infty} M_{n} \neq \varphi$ and $\omega\left\{\bigcap_{k=1}^{n} M_{n}\right\}=0$, i.e., $\bigcap_{k=1}^{\infty} M_{n}$ is relatively weakly compact.

The map $\omega\{\cdot\}$ is called the De Blasi measure of weak noncompactness. In [9], Appell and De Pascale proved that in $L^{1}$-spaces $\omega\{\cdot\}$ has the following form:

$$
\omega\{M\}=\limsup _{\varepsilon \rightarrow 0}\left\{\sup _{\psi \in M}\left[\int_{D}\|\psi(t)\|_{X} d t: \operatorname{meas}(D) \leq \varepsilon\right]\right\}
$$

for all bounded subsets $M$ of $L^{1}(\Omega ; X)$, where $X$ is a finite dimensional Banach space and meas $(\cdot)$ denotes the Lebesgue measure. 
Throughout this paper, $X$ denotes a Banach space; $\mathcal{D}(T)$ and $\mathcal{R}(T)$, respectively, denote the domain and range of operator $T$; and $\omega\{S\}$ denotes the De Blasi measure of weak noncompactness of bounded subset $S$.

Definition 2.2 An operator $T: \mathcal{D}(T) \subset X \rightarrow X$ is said to be $\omega$-contractive (or a $\omega$ - $\alpha$ contraction) if it maps bounded sets into bounded sets, and there exists some $\alpha \in[0,1)$ such that $\omega\{T(S)\} \leq \alpha \omega\{S\}$ for all bounded sets $S$ in $\mathcal{D}(T)$.

An operator $T: \mathcal{D}(T) \subset X \rightarrow X$ is said to be $\omega$-condensing if it maps bounded sets into bounded sets, and $\omega\{T(S)\}<\omega\{S\}$ for all bounded sets $S$ in $\mathcal{D}(T)$ with $\omega\{S\}>0$.

Remark 2.3 Obviously, every $\omega$ - $\alpha$-contraction with $0 \leq \alpha<1$ is $\omega$-condensing.

Let $T$ be an operator from $\mathcal{D}(T) \subset X$ into $X$. Latrach et al. [7] introduce the following conditions:

$(\mathcal{A} 1)$ If $\left(x_{n}\right)_{n \in \mathbb{N}}$ is a weakly convergent sequence in $\mathcal{D}(T)$, then $\left(T x_{n}\right)_{n \in \mathbb{N}}$ has a strongly convergent subsequence in $X$.

$(\mathcal{A} 2)$ If $\left(x_{n}\right)_{n \in \mathbb{N}}$ is a weakly convergent sequence in $\mathcal{D}(T)$, then $\left(T x_{n}\right)_{n \in \mathbb{N}}$ has a weakly convergent subsequence in $X$.

The conditions $(\mathcal{A} 1)$ and $(\mathcal{A} 2)$ were already considered in $[7,8,10-14]$.

\section{Remark 2.4}

(a) Operators satisfying either $(\mathcal{A} 1)$ or $(\mathcal{A} 2)$ are not necessarily weakly continuous.

(b) An operator satisfies $(\mathcal{A} 1)$ if and only if it maps relatively weakly compact sets into relatively strongly compact ones.

(c) An operator satisfies $(\mathcal{A} 2)$ if and only if it maps relatively weakly compact sets into relatively weakly compact ones (Eberlein-Šmulian theorem, see, e.g.,

[15, pp.248-250]).

(d) Every $\omega$-contractive operator satisfies $(\mathcal{A} 2)$.

(e) The condition $(\mathcal{A} 2)$ holds true for every bounded linear operator.

Lemma 2.5 Let operator $T: \mathcal{D}(T) \subset X \rightarrow X$ satisfy $(\mathcal{A} 1)$, and let operator $Q: \mathcal{R}(T) \subset$ $X \rightarrow X$ be continuous. Then the compound operator $Q \circ T: \mathcal{D}(T) \subset X \rightarrow X$ satisfies $(\mathcal{A} 1)$.

Proof Let $\left(x_{n}\right)_{n \in \mathbb{N}}$ be a weakly convergent sequence in $\mathcal{D}(T)$. By the hypothesis of $T$ satisfying $(\mathcal{A} 1),\left(T x_{n}\right)_{n \in \mathbb{N}}$ has a strongly convergent subsequence, say $\left(T x_{n_{k}}\right)_{k \in \mathbb{N}}$. The continuity of $Q$ implies that $\left(Q T x_{n_{k}}\right)_{k \in \mathbb{N}}$ is also strongly convergent, and therefore $Q \circ T$ satisfies $(\mathcal{A} 1)$.

Definition 2.6 An operator $T: \mathcal{D}(T) \subset X \rightarrow X$ is said to be $\varphi$-nonlinear contractive (or an $\varphi$-nonlinear contraction), if there exists a continuous and nondecreasing function $\varphi$ : $\mathbb{R}^{+} \rightarrow \mathbb{R}^{+}$such that

$$
\|T x-T y\| \leq \varphi(\|x-y\|) \quad \text { for all } x, y \in \mathcal{D}(T)
$$

where $\varphi(r)<r$ for $r>0$.

Remark 2.7 Obviously, all strictly contractions are $\varphi$-nonlinear contractions. 
Lemma 2.8 (see [16, Lemma 1.11]) Let operator $T: \mathcal{D}(T) \subset X \rightarrow X$ be $\varphi$-nonlinear contractive on a Banach space $X$ and satisfy $(\mathcal{A} 2)$. Then for each bounded subset $M$ of $X$ one has $\omega\{T(M)\} \leq \varphi(\omega\{M\})$.

Lemma 2.9 If an operator $T: X \rightarrow X$ is $\varphi$-nonlinear contractive, then $F:=I-T$ is a homeomorphism of $X$ onto $X$.

Proof For any $y \in X$, we define the operator $\tau_{y}$ from $X$ to $X$, by $\tau_{y} x=T x+y$. Since $T$ is $\varphi$-nonlinear contractive, it is easy to see that $\tau_{y}$ is also $\varphi$-nonlinear contractive. According to Theorem 1 in [17], $\tau_{y}$ has a unique fixed point $x$ such that $x=T x+y$, i.e. $y=(I-T) x$, and then $F:=I-T$ is surjective on $X$.

If $x, y \in X$ and $x \neq y$, then

$$
\|F x-F y\|=\|(x-T x)-(y-T y)\| \geq\|x-y\|-\|T x-T y\| \geq\|x-y\|-\varphi(\|x-y\|)>0,
$$

which implies that $F$ is injective and $F^{-1}$ exists on $X$.

For proving the continuity of $F^{-1}$, suppose that there exists a point $x$ and a sequence $\left(x_{n}\right)_{n \in \mathbb{N}}$ in $X$ such that $F x_{n} \rightarrow F x$, and $\lim _{n \rightarrow \infty} \sup _{k \geq n}\left\|x_{k}-x\right\|=a$. Consequently, from the inequality

$$
\left\|F x_{n}-F x\right\| \geq\left\|x_{n}-x\right\|-\varphi\left(\left\|x_{n}-x\right\|\right)
$$

we obtain that $0 \geq a-\varphi(a)$, which implies that $a=0$ and, therefore, $F^{-1}$ is continuous.

\section{Fixed-point theorems for the sum of two operators}

The following theorem was proved by Ben Amar and Garcia-Falset [13], and its more general form was presented by Agarwal et al. [14] is a variant of the Sadovskii fixed-point theorem for the classes of operators which satisfy $(\mathcal{A} 1)$.

Theorem 3.1 (see [13, Theorem 3.1] or [14, Theorem 2.1 and Corollary 2.2]) Let $M$ be a nonempty, bounded, closed and convex subset of a Banach space X. Assume that $T: M \rightarrow$ $M$ is continuous and satisfies (A1). If $T$ is $\omega$-condensing, then it has a fixed point in $M$.

Our purpose here is to establish a fixed-point theorem for the sum of a $\omega$-contractive operator and an $\varphi$-nonlinear contractive operator.

Theorem 3.2 Let $M$ be a nonempty, bounded, closed and convex subset of a Banach space $X$. Suppose that $A: M \rightarrow X$ and $B: X \rightarrow X$ are two operators such that

(i) $A$ is a continuous $\omega$ - $\alpha$-contraction with $\alpha \in[0,1)$, and $A$ satisfies $(\mathcal{A} 1)$,

(ii) $B$ is an $\varphi$-nonlinear contraction with $\varphi(r)<(1-\alpha) r$ for $r>0$, and $B$ satisfies $(\mathcal{A} 2)$,

(iii) $(x=B x+A y, y \in M) \Rightarrow x \in M$.

Then there is a point $x \in M$ such that $A x+B x=x$.

Proof By Lemma 2.9, $I-B$ has a continuous inverse defined on $X$, and then $J:=(I-B)^{-1} A$ is well defined on $M$. Once we prove that $J$ has a fixed point in $M$, the proof is achieved. 
For any $y \in M$, according to Lemma 2.9 there exists a unique $x \in X$ such that $A y=x-B x$. The hypothesis (iii) shows that $x \in M$, which implies that $A(M) \subset(I-B)(M)$ and, therefore, $J(M) \subset M$.

Obviously, the compound operator $J$ is continuous since $A$ and $(I-B)^{-1}$ is continuous and by Lemma $2.5, J$ satisfies $(\mathcal{A} 1)$. Now by referring to the formula

$$
\left.(I-B)^{-1} A=A+B(I-B)^{-1} A \quad \text { (i.e., } J=A+B J\right),
$$

for every subset $S$ of $M$ with $\omega\{S\}>0$, we have

$$
J(S) \subset A(S)+B J(S) .
$$

Since $A$ is $\omega$ - $\alpha$-contractive and $B$ satisfies $\omega\{B J(S)\} \leq \varphi(\omega\{J(S)\})$ by Lemma 2.8, we have

$$
\omega\{J(S)\} \leq \omega\{A(S)\}+\omega\{B J(S)\} \leq \alpha \omega\{S\}+\varphi(\omega\{J(S)\}) .
$$

Now, if $\alpha=0$, inequality (3.1) becomes $\omega\{J(S)\} \leq \varphi(\omega\{J(S)\})$, which implies that $\omega\{J(S)\}=0$. Otherwise, by recalling the assumption that $\varphi(r)<(1-\alpha) r$ for $r>0$, inequality (3.1) becomes

$$
\omega\{J(S)\}<\alpha \omega\{S\}+(1-\alpha) \omega\{J(S)\}, \quad \text { i.e., } \omega\{J(S)\}<\omega\{S\} .
$$

In both cases, $J$ is shown to be $\omega$-condensing. Now the use of Theorem 3.1 achieves the proof.

Remark 3.3 It should be noticed to the following particular cases:

(1) If we take $B=0$, then we return the above theorem back to [7, Theorem 2.2], which is an extension of the Darbo fixed-point theorem for $\omega$-contractive operators.

(2) If we take $\alpha=0$ and the function $\varphi(r)=\beta r(0 \leq \beta<1)$ in the above theorem, we obtain a result which was [7, Theorem 2.3].

(3) If we only take the function $\varphi(r)=\beta r(0 \leq \beta<1-\alpha)$ in the above theorem, we obtain the following Corollary 3.4, which is a new fixed-point theorem for the sum of two operators.

(4) If we only take $\alpha=0$ in the above theorem, we obtain the following Corollary 3.6, which is the new version of Krasnosel'skii-type fixed-point theorems.

Corollary 3.4 Let $M$ be a nonempty, bounded, closed and convex subset of a Banach space $X$. Suppose that $A: M \rightarrow X$ and $B: X \rightarrow X$ are two operators such that

(i) $A$ is a continuous $\omega$ - $\alpha$-contraction with $\alpha \in[0,1)$, and $A$ satisfies $(\mathcal{A} 1)$,

(ii) $B$ is a strict contraction with $\beta \in[0,1-\alpha)$, and $B$ satisfies $(\mathcal{A} 2)$,

(iii) $(x=B x+A y, y \in M) \Rightarrow x \in M$.

Then there is a point $x \in M$ such that $A x+B x=x$.

Remark 3.5 The above corollary is a variant and supplement of [6, Theorem 3.3], in which the authors demand that the operators $A$ and $B$ are weakly sequentially continuous. 
Corollary 3.6 Let $M$ be a nonempty, bounded, closed and convex subset of a Banach space $X$. Suppose that $A: M \rightarrow X$ and $B: X \rightarrow X$ are two operators such that

(i) $A$ is a continuous, $A(M)$ is relatively weakly compact and $A$ satisfies $(\mathcal{A} 1)$,

(ii) $B$ is an $\varphi$-nonlinear contraction, and $B$ satisfies $(\mathcal{A} 2)$,

(iii) $(x=B x+A y, y \in M) \Rightarrow x \in M$.

Then there is a point $x \in M$ such that $A x+B x=x$.

Remark 3.7 The above corollary is a variant and supplement of [18, Theorem 2.1], in which the author demands that the operators $A$ and $B$ are weakly sequentially continuous, and $B$ is strictly contractive.

Now, on the basis of Corollary 3.6, we prove the following fixed-point theorem for the sum of a weakly-strongly continuous operator and a nonexpansive operator.

Theorem 3.8 Let $M$ be a nonempty, bounded, closed and convex subset of a Banach space $X$. Suppose that $A: M \rightarrow X$ and $B: X \rightarrow X$ are two operators such that

(i) $A$ is weakly-strongly continuous, and $A(M)$ is relatively weakly compact,

(ii) $B$ is nonexpansive and $\omega$-condensing,

(iii) $I-B$ is demiclosed,

(iv) if $\lambda \in(0,1)$ and $x=\lambda B x+$ Ay for some $y \in M$, then $x \in M$.

Then there is a point $x \in M$ such that $A x+B x=x$.

\section{Remark 3.9}

(1) Recall that an operator $T: \mathcal{D}(T) \subset X \rightarrow X$ is said to be demiclosed if for any sequence $\left(x_{n}\right)_{n \in \mathbb{N}}$ in $D(T)$ that $x_{n} \rightarrow x$ and $T x_{n} \rightarrow y$, then $x \in \mathcal{D}(T)$ and $T x=y$.

(2) The assumption (iv) in the above theorem was first introduced in [19], it is slight different with [10, Corollary 3.1] and [11, Theorem 2.1].

(3) In [11, Theorem 2.1] and [18, Theorem 2.4], the following condition is required: if $\left(x_{n}\right)_{n \in \mathbb{N}}$ is a sequence of $M$ such that $\left(x_{n}-T x_{n}\right)_{n \in \mathbb{N}}$ is weakly convergent, then $\left(x_{n}\right)_{n \in \mathbb{N}}$ has a weakly convergent subsequence. In the above theorem, we replaced it with the $\omega$-condensing of $B$.

Proof of Theorem 3.8 For each $\lambda \in(0,1)$, the operators $A$ and $\lambda B$ fulfill the conditions of Corollary 3.6 and, therefore, there is a point $x_{\lambda} \in M$ such that $x_{\lambda}=\lambda B x_{\lambda}+A x_{\lambda}$. Now choose a sequence $\left(\lambda_{n}\right)_{n \in \mathbb{N}} \subset(0,1)$ such that $\lambda_{n} \rightarrow 1$. Consequently, there exists a sequence $\left(x_{n}\right)_{n \in \mathbb{N}} \subset M$ such that

$$
x_{n}=\lambda_{n} B x_{n}+A x_{n} .
$$

Let $S=\left\{x_{n}: n \in \mathbb{N}\right\}$. We claim that $S$ is relatively weakly compact. Suppose that it is not the case, by assumption (i) and (ii), we have

$$
\begin{aligned}
\omega\{S\} & =\omega\left\{x_{n}: n \in \mathbb{N}\right\}=\omega\left\{\lambda_{n} B x_{n}+A x_{n}: n \in \mathbb{N}\right\} \\
& \leq \lambda_{n} \omega\{B(S)\}+\omega\{A(S)\}=\lambda_{n} \omega\{B(S)\}<\omega\{S\} .
\end{aligned}
$$

This contradiction tells us that the sequence $\left(x_{n}\right)_{n \in \mathbb{N}}$ has a weakly convergent subsequence, i.e., there exists $\left(x_{n_{k}}\right)_{k \in \mathbb{N}}$ such that $x_{n_{k}} \rightarrow x \in M$. By assumption (i), we have $A x_{n_{k}} \rightarrow A x$, 
and then $\left(I-\lambda_{n_{k}} B\right) x_{n_{k}} \rightarrow A x$. Since $\left(x_{n_{k}}\right)_{k \in \mathbb{N}}$ is contained in bounded set $M$, and $B$ maps $M$ into a bounded set ( $B$ is nonexpansive), then $\left\|B x_{n_{k}}\right\|$ is norm bounded. Thus, we have $\left(1-\lambda_{n_{k}}\right)\left\|B x_{n_{k}}\right\| \rightarrow 0$. Moreover, we have

$$
\begin{aligned}
\left\|(I-B) x_{n_{k}}-A x\right\| & \leq\left\|(I-B) x_{n_{k}}-\left(I-\lambda_{n_{k}} B\right) x_{n_{k}}\right\|+\left\|\left(I-\lambda_{n_{k}} B\right) x_{n_{k}}-A x\right\| \\
& =\left(1-\lambda_{n_{k}}\right)\left\|B x_{n_{k}}\right\|+\left\|\left(I-\lambda_{n_{k}} B\right) x_{n_{k}}-A x\right\| \rightarrow 0,
\end{aligned}
$$

that is, $(I-B) x_{n_{k}} \rightarrow A x$. By assumption (iii), we have $(I-B) x=A x$, and then the proof is achieved.

If the Banach space $X$ is reflexive, then $B$ is always $\omega$-condensing on $M$ (see, e.g., [15, p.251]). Moreover, if we supposed that $X$ is uniformly convex Banach space, then $I-B: M \rightarrow X$ is demiclosed (see, e.g., [20, pp.476-478]). Thus, we obtain the following consequence.

Corollary 3.10 Let $M$ be a nonempty, bounded, closed and convex subset of a uniformly convex Banach space $X$. Suppose that $A: M \rightarrow X$ and $B: X \rightarrow X$ are two operators such that

(i) A is weakly-strongly continuous,

(ii) $B$ is nonexpansive,

(iii) if $\lambda \in(0,1)$ and $x=\lambda B x+$ Ay for some $y \in M$, then $x \in M$.

Then there is a point $x \in M$ such that $A x+B x=x$.

In order to use the above results on the whole space, we first prove the following result.

Theorem 3.11 Let $X$ be a Banach space $X$. Assume that the operator $T: X \rightarrow X$ be continuous $\omega$-condensing and satisfies (A1). Then either

(a) equation $x=T x$ has a solution, or

(b) the set $\{x \in X: x=\lambda T(x)\}$ is unbounded for some $\lambda \in(0,1)$.

Proof Choose an arbitrary $R>0$. Define for each $x \in X$

$$
\rho(x)= \begin{cases}x, & \|x\| \leq R, \\ \frac{R}{\|x\|} x, & \|x\|>R .\end{cases}
$$

Clearly, $\rho$ is a continuous retraction of $X$ on $\mathbf{B}_{R}$. Thus, we can define the mapping $T_{\rho}$ : $\mathbf{B}_{R} \rightarrow \mathbf{B}_{R}$ by $T_{\rho} x=\rho(T x)$.

Since $T$ and $\rho$ are continuous, obviously $T_{\rho}$ is also continuous. Furthermore, since $T$ satisfies $(\mathcal{A} 1)$ and $\rho$ is continuous, hence $T_{\rho}$ also satisfies $(\mathcal{A} 1)$. We next claim that $\omega\left\{T_{\rho}(S)\right\}<\omega\{S\}$ for any $S \subset \mathbf{B}_{r}$ with $\omega\{S\}>0$.

Indeed, $T_{\rho}\left(\mathbf{B}_{r}\right)=\rho\left(T\left(\mathbf{B}_{r}\right)\right)$. For any $x \in \mathbf{B}_{r}$, there are two possibilities:

(1) $\|T x\| \leq R$; in this case, $\rho(T x)=T x \in T(S) \subset \operatorname{co}(T(S) \cup\{\mathbf{0}\})$.

(2) $\|T x\|>R$; in this case, $\rho(T x)=\frac{R}{\|T x\|} T x=\frac{R}{\|T x\|} T x+\left(1-\frac{R}{\|T x\|}\right) \cdot \mathbf{0} \in \operatorname{co}(T(S) \cup\{\mathbf{0}\})$.

The above argument yields $T_{\rho}(S) \subset \operatorname{co}(T(S) \cup\{\mathbf{0}\})$. 
Now, by using the properties of the measure of weak noncompactness and properties of $T$, we have that

$$
\omega\left\{T_{\rho}(S)\right\} \leq \omega\{T(S)\}<\omega\{S\}
$$

as claimed, that is, $T_{\rho}$ is a $\omega$-condensing.

The above argument shows that $T_{\rho}: \mathbf{B}_{r} \rightarrow \mathbf{B}_{r}$ is under the conditions of Theorem 3.1 and thus we have that there exists $x_{0} \in \mathbf{B}_{r}$ such that $x_{0}=T_{\rho} x_{0}$. Indeed, we obtain the following results:

(a) if $T x_{0} \in \mathbf{B}_{R}$, then $x_{0}=T_{\rho} x_{0}=\rho\left(T x_{0}\right)=T x_{0}$, that is, $T$ has a fixed point; otherwise,

(b) if $T x_{0} \notin \mathbf{B}_{R}$, then $x_{0}=T_{\rho} x_{0}=\rho\left(T x_{0}\right)=\frac{R}{\left\|T x_{0}\right\|} T x_{0}$, that is, $x_{0}$ is a solution of the equation $x=\lambda T x$ for $\lambda=\frac{R}{\left\|T x_{0}\right\|} \in(0,1)$ and $\left\|x_{0}\right\|=R$.

Consequently, if there is no $x \in \mathbf{B}_{R}$ such that $x=T x$ for any $R>0$, then the above arguments show that the set of solutions of equation $x=\lambda T(x)$ is unbounded for some $\lambda \in(0,1)$.

We are now in a position to prove the main result on the whole space.

Theorem 3.12 Let $X$ be a Banach space. Suppose that $A, B: X \rightarrow X$ are two operators such that

(i) $A$ is a continuous $\omega$ - $\alpha$-contraction with $\alpha \in[0,1)$, and $A$ satisfies $(\mathcal{A} 1)$,

(ii) $B$ is an $\varphi$-nonlinear contraction with $\varphi(r)<(1-\alpha) r$ for $r>0$, and $B$ satisfies $(\mathcal{A} 2)$,

(iii) function $\varphi$ satisfies $\lim _{r \rightarrow+\infty}[r-\varphi(r)]=+\infty$.

Then, either

(a) the equation $x=A x+B x$ has a solution, or

(b) the set $\{x \in X: x=\lambda B(x / \lambda)+\lambda A(x)\}$ is unbounded for some $\lambda \in(0,1)$.

Remark 3.13 Obviously, assumption (iii) in the above theorem is unnecessary whenever $\alpha \neq 0$.

Proof of Theorem 3.12 As in the proof of Theorem 3.2, it can be seen that the compound operator $J:=(I-B)^{-1} A$ is well defined from $X$ into $X$. Clearly, $J$ is continuous and $J$ satisfies $(\mathcal{A} 1)$ by Lemma 2.5 .

Let us prove that $J$ maps bounded set into a bounded set. For any bounded set $S$ such that $u, v \in(I-B)^{-1} A(S)$, there exist $x, y \in S$ such that $u=(I-B)^{-1} A x$ and $v=(I-B)^{-1} A y$, that is, $u-B u=A x$ and $v-B v=A y$. Thus, by assumption (ii) and the boundness of $A(S)$, we have

$$
\|u-v\|-\varphi(\|u-v\|) \leq\|A x-A y\| \leq \operatorname{diam}(A(S))<+\infty .
$$

Suppose that $J(S)=(I-B)^{-1} A(S)$ is unbounded, i.e., there exist sequences $\left(u_{n}\right)_{n \in \mathbb{N}}$ and $\left(v_{n}\right)_{n \in \mathbb{N}}$ such that $\left\|u_{n}-v_{n}\right\| \rightarrow+\infty$, and then $\left\|u_{n}-v_{n}\right\|-\varphi\left(\left\|u_{n}-v_{n}\right\|\right) \rightarrow+\infty$ by assumption (iii). This is a contradiction with $\operatorname{diam}(A(S))<+\infty$ in (3.2) and, therefore, $J(S)$ is bounded.

It is similar to that of Theorem 3.2 to prove that $\omega\{J(S)\}<\omega\{S\}$ for every bounded set $S$ in $\mathcal{D}(T)$ with $\omega\{S\}>0$. Now, by using Theorem 3.11 for operator $J$, we obtain that either 
(a) the equation $x=A x+B x$ has a solution which is the solution of equation $x=J x$, or

(b) the set $\{x \in X: x=\lambda B(x / \lambda)+\lambda A(x)\}=\left\{x \in X: x=\lambda(I-B)^{-1} A x\right\}$ is unbounded for some $\lambda \in(0,1)$.

Corollary 3.14 Let $X$ be a Banach space. Suppose that $A, B: X \rightarrow X$ are two operators such that

(i) $A$ is a continuous $\omega$ - $\alpha$-contraction with $\alpha \in[0,1)$, and $A$ satisfies $(\mathcal{A} 1)$,

(ii) $B$ is a strict contraction with $\beta \in[0,1-\alpha)$, and $B$ satisfies $(\mathcal{A} 2)$.

Then, either

(a) the equation $x=A x+B x$ has a solution, or

(b) the set $\{x \in X: x=\lambda B(x / \lambda)+\lambda A(x)\}$ is unbounded for some $\lambda \in(0,1)$.

Corollary 3.15 Let $X$ be a Banach space. Suppose that $A, B: X \rightarrow X$ are two operators such that

(i) $A$ is a continuous, $A(M)$ is relatively weakly compact, and $A$ satisfies $(\mathcal{A} 1)$,

(ii) $B$ is an $\varphi$-nonlinear contraction, and $B$ satisfies $(\mathcal{A} 2)$,

(iii) function $\varphi$ satisfies $\lim _{r \rightarrow+\infty}[r-\varphi(r)]=+\infty$.

Then, either

(a) the equation $x=A x+B x$ has a solution, or

(b) the set $\{x \in X: x=\lambda B(x / \lambda)+\lambda A(x)\}$ is unbounded for some $\lambda \in(0,1)$.

\section{Application to Hammerstein integral equations in $L^{1}$ space}

Let $\Omega$ be a domain of $\mathbb{R}^{n}$. A function $f: \Omega \times X \rightarrow Y$ is said to be a Carathéodory function if

(i) for any fixed $x \in X$, the function $t \rightarrow f(t, x)$ is measurable from $\Omega$ to $Y$;

(ii) for almost any $t \in \Omega$, the function $f(t, \cdot): X \rightarrow Y$ is continuous.

Let $m(\Omega)$ be the set of all measurable functions $\psi: \Omega \rightarrow X$. If $f$ is a Carathéodory function, then $f$ defines an operator $\mathbf{N}_{f}: m(\Omega) \rightarrow m(\Omega)$ by $\mathbf{N}_{f}(\psi)(t) \rightarrow f(t, \psi(t))$. This operator is called the Nemytskii operator associated to $f$ (or the superposition operator). Regarding its continuity and weak compactness, we have the following lemma.

Lemma 4.1 Let $X, Y$ be two finite dimensional Banach spaces. If $f: \Omega \times X \rightarrow Y$ is a Carathéodory function, then the Nemytskii operator $\mathbf{N}_{f}$ maps $L^{1}(\Omega ; X)$ into $L^{1}(\Omega ; Y)$ if and only if there exist a constant $b \geq 0$ and a function $a \in L_{+}^{1}(\Omega)$ such that

$$
\|f(t, x)\|_{Y} \leq a(t)+b\|x\|_{X}
$$

where $L_{+}^{1}(\Omega)$ denotes the positive cone of the space $L^{1}(\Omega)($ see $[21]$ or $[22])$.

With the conditions of Lemma 4.1, the operator $\mathbf{N}_{f}$ is obviously continuous and maps bounded sets of $L^{1}(\Omega ; X)$ into bounded sets of $L^{1}(\Omega ; Y)$.

Lemma 4.2 (see [8, Lemma 3.2]) Let $\Omega$ be a bounded domain in $\mathbb{R}^{n}$. Iff $: \Omega \times X \rightarrow Y$ is a Carathéodory function and $\mathbf{N}_{f}$ maps $L^{1}(\Omega ; X)$ into $L^{1}(\Omega ; Y)$, then $\mathbf{N}_{f}$ satisfies $(\mathcal{A} 2)$.

Remark 4.3 Although Nemytskii operator $\mathbf{N}_{f}$ satisfies $(\mathcal{A} 2)$, generally it is not weakly continuous. In fact, only linear functions generate weakly continuous Nemytskii operators 
in $L^{1}$ spaces (see, for instance, [9, Theorem 2.6]). The question of considering the weak sequential continuity of the Nemytskii operator acting from space $L^{p}$ to space $L^{q}(1 \leq p, q<$ $\infty)$ is discussed in [22] and the answer is shown to be negative at least for $p=2$.

Next, we give an example of application for Theorem 3.12 in the Banach space of integrable function $L^{1}(\Omega ; X)$.

Example 4.4 We will study now the existence of solutions for the following variant of Hammerstein's integral equation

$$
\psi(t)=g(t, \psi(s))+\mu \int_{\Omega} k(t, s) f(s, \psi(s)) d s,
$$

in $L^{1}(\Omega ; X)$, the space of Lebesgue integrable functions on a measurable subset $\Omega$ of $\mathbb{R}^{n}$ with values in a finite dimensional Banach space $X$. Here, $f$ is a nonlinear function and $k$ is measurable, while $g$ is a function satisfying $\varphi$-nonlinear contractive condition in $L^{1}(\Omega ; X)$.

First, observe that the above problem may be written in the form

$$
\psi=A \psi+B \psi
$$

where $B$ is the Nemytskii operator associated to the function $g\left(\right.$ i.e., $\left.B=\mathbf{N}_{g}\right)$ from $L^{1}(\Omega ; X)$ into $L^{1}(\Omega ; X)$ by

$$
B \psi(t)=g(t, \psi(t))
$$

and $A=\mu L \mathbf{N}_{f}$ is the product of the Nemytskii operator associated to $f$ and the linear integral operator $\mu L$ where $\mu \in \mathbb{C}$ and $L$ is defined from $L^{1}(\Omega ; Y)$ into $L^{1}(\Omega ; X)$ by

$$
(L \psi)(t)=\int_{\Omega} k(t, s) \psi(s) d s
$$

Let us now introduce the following assumptions.

\section{Assumptions 4.5}

(a) $f$ is a Carathéodory function and $\mathbf{N}_{f}$ acts from $L^{1}(\Omega ; X)$ into $L^{1}(\Omega ; Y)$;

(b) $b|\mu|\|L\|<1$ (the constant $b$ was introduced in Lemma 4.1);

(c) $g: \Omega \times X \rightarrow X$ is a measurable function with $g(\cdot, 0) \in L^{1}(\Omega ; X)$, and there exists a continuous and nondecreasing function $\varphi: \mathbb{R}^{+} \rightarrow \mathbb{R}^{+}$such that

$$
\int_{\Omega}\|g(t, x)-g(t, y)\|_{X} d t \leq \varphi\left(\|x-y\|_{L^{1}(\Omega ; X)}\right) \quad \text { for all } x, y \in X,
$$

where $\varphi(r)<(1-b|\mu|\|L\|) r$ for $r>0$ and $\lim _{r \rightarrow+\infty}[r-\varphi(r)]=+\infty$;

(d) the function $k: \Omega \times \Omega \rightarrow L(Y, X)$ is strongly measurable and the linear operator $L$ defined by (4.2) maps $L^{1}(\Omega ; Y)$ into $L^{1}(\Omega ; X)$;

(e) the functions $\rho(t): \Omega \rightarrow L(Y, X), s \rightarrow \rho(t)(s):=k(t, s)$ belong to $L^{\infty}(\Omega ; L(Y, X))$; 
(f) the solution of the integral equation

$$
\psi(t)=\lambda g\left(t, \frac{1}{\lambda} \psi(t)\right)+\lambda \mu \int_{\Omega} k(t, s) f(s, \psi(s)) d s
$$

is bounded for $\lambda \in(0,1)$.

Remark 4.6 (1) Clearly, the condition that $\lim _{r \rightarrow+\infty}[r-\varphi(r)]=+\infty$ in assumption (c) is unnecessary whenever $b|\mu||| L \| \neq 0$;

(2) It should be noted that assumptions (d) and (e) lead to the estimate

$$
\left\|\int_{\Omega} k(t, s) x(s) d s\right\| \leq\|\rho\|_{L^{\infty}(\Omega ; L(Y, X))}\|x\|_{L^{1}(\Omega ; Y)},
$$

and so

$$
\|L \psi\|=\int_{\Omega}\left\|\int_{\Omega} k(t, s) \psi(s) d s\right\|_{X} d t \leq\|\rho\|_{L^{1}\left(\Omega ; L^{\infty}\right)}\|\psi\|_{L^{1}(\Omega ; Y)} .
$$

This shows that the linear operator $L$ is continuous, hence weakly continuous from $L^{1}(\Omega ; Y)$ into $L^{1}(\Omega ; X)$ and that $\|L\| \leq\|\rho\|_{L^{1}\left(\Omega ; L^{\infty}\right)}$.

(3) By assumption (c), we get

$$
\int_{\Omega}\|g(t, u)\|_{X} d t \leq \int_{\Omega}\|g(t, 0)\|_{X} d t+\varphi\left(\|u\|_{X}\right)<\int_{\Omega}\|g(t, 0)\|_{X} d t+(1-b|\lambda|\|L\|)\|u\|_{X},
$$

for every $u \in X$. This shows that the Nemytskii operator $\mathbf{N}_{g}$ is continuous and maps bounded sets of $L^{1}(\Omega ; X)$ into bounded sets of $L^{1}(\Omega ; X)$. According to Lemma 4.2, the operator $B$ satisfies $(\mathcal{A} 2)$.

Now we are in a position to state our main result.

Theorem 4.7 Let $X$ and $Y$ be two finite dimensional Banach spaces and $\Omega$ be a bounded domain of $\mathbb{R}^{n}$. Assume that the conditions (a)-(f) are satisfied, then the problem (4.1) has at least one solution in $L^{1}(\Omega ; X)$.

Proof Let us first observe that Lemma 4.1 implies that there are $a \in L_{+}^{1}(\Omega)$ and $b>0$ such that

$$
\|f(t, x)\|_{Y} \leq a(t)+b\|x\|_{X}
$$

So, for any bounded subset $S$ of $L^{1}(\Omega ; X)$, we have

$$
\int_{S}\left\|\mathbf{N}_{f} \psi(t)\right\|_{Y} d t \leq \int_{S} a(t) d t+b \int_{S}\|\psi(t)\|_{X} d t
$$

According to (2.1), this leads $\omega\left\{\mathbf{N}_{f}(S)\right\} \leq b \omega\{S\}$. Thus, we have

$$
\omega\{A(S)\}=\omega\left(\mu L \mathbf{N}_{f}(S)\right) \leq b|\mu|\|L\| \omega(S)
$$

which implies that $A$ is a $\omega-b|\mu|\|L\|$-contractive. 
On the other hand, clearly $A$ is continuous (see Lemma 4.1 and Remark 4.6(2)). Now we check that $A$ satisfies the condition $(\mathcal{A} 1)$. For this end, let $\left(x_{n}\right)_{n \in \mathbb{N}}$ be a weakly convergent sequence of $L^{1}(\Omega ; X)$. Using the fact that $\mathbf{N}_{f}$ satisfies $(\mathcal{A} 2)$, and then $\left(\mathbf{N}_{f} x_{n}\right)_{n \in \mathbb{N}}$ has a weakly convergent subsequence, say $\left(\mathbf{N}_{f} x_{n_{k}}\right)_{k \in \mathbb{N}}$. Moreover, the continuity of the linear operator $L$ implies its weak continuity on $L^{1}(\Omega ; Y)$. Thus, the sequence $\left(L \mathbf{N}_{f} x_{n_{k}}\right)_{k \in \mathbb{N}}$, i.e. $\left(A x_{n_{k}}\right)_{k \in \mathbb{N}}$ converges pointwisely for a.e. $t \in \Omega$. Using Vitali's convergence theorem, we conclude that $\left(A x_{n_{k}}\right)_{k \in \mathbb{N}}$ converges strongly in $L^{1}(\Omega ; X)$. Therefore, $A$ satisfies $(\mathcal{A} 1)$.

Let $x(t), y(t) \in L^{1}(\Omega ; X)$. It follows from assumption (c) that

$$
\|B x-B y\|_{L^{1}(\Omega ; X)}=\int_{\Omega}\|g(t, x(t))-g(t, y(t))\|_{X} d t \leq \varphi\left(\|x(t)-y(t)\|_{L^{1}(\Omega ; X)}\right) .
$$

So, $B$ is $\varphi$-nonlinear contractive on $L^{1}(\Omega ; X)$ and from Remark 4.6(3), $B$ satisfies $(\mathcal{A} 2)$.

The above arguments show that $A$ and $B$ satisfy the conditions of Theorem 3.12, and assumption (f) allows us to affirm that equation (4.1) has a solution.

Remark 4.8 Equation (4.1) was respectively considered in [8] and [12] under different assumptions. However, our results relax some assumptions of [8, Theorem 3.1] and [12, Theorem 3.1].

\section{Competing interests}

The author declares that he has no competing interests.

\section{Acknowledgements}

The author is grateful to the referee for the careful reading of the manuscript. They brought to my attention a proof of Theorem 3.1, which had been published in [13] and [14]; also, the remarks motivated the author to make several other valuable improvements.

Received: 20 September 2012 Accepted: 4 April 2013 Published: 18 April 2013

\section{References}

1. Krasnosel'skii, MA: Some problems of nonlinear analysis. Am. Math. Soc. Transl. Ser. 2 10(2), 345-409 (1958)

2. Darbo, G: Punti uniti in transformazioni a codominio non compatto. Rend. Semin. Mat. Univ. Padova 24, 84-92 (1955)

3. Sadovskii, BN: On a fixed point principle. Funkc. Anal. Prilozh. 4(2), 74-76 (1967)

4. De Blasi, FS: On a property of the unit sphere in Banach spaces. Bull. Math. Soc. Sci. Math. Roum. 21, $259-262$ (1977)

5. Emmanuele, G: Measure of weak noncompactness and fixed point theorems. Bull. Math. Soc. Sci. Math. Roum. 25, 353-358 (1981)

6. Garcia-Falset, J, Latrach, K: Krasnosel'skii-type fixed-point theorems for weakly sequentially continuous mappings. Bull. Lond. Math. Soc. 44, 25-38 (2012)

7. Latrach, K, Taoudi, MA, Zeghal, A: Some fixed point theorems of the Schauder and Krasnosel'skii type and application to nonlinear transport equations. J. Differ. Equ. 221, 256-271 (2006)

8. Latrach, K, Taoudi, MA: Existence results for a generalized nonlinear Hammerstein equation on $L^{1}$-spaces. Nonlinear Anal. 66, 2325-2333 (2007)

9. Appell, J, De Pascale, E: Su alcuni parametri connessi con la misura di non compattezza di Hausdorff in spazi di funzioni misurabili. Boll. Unione Mat. Ital, B 3, 497-515 (1984)

10. Garcia-Falset, J, Latrach, K, Moreno-Galvez, E, Taoudi, MA: Schaefer-Krasnosel'skii fixed point theorems using a usual measure of weak noncompactness. J. Differ. Equ. 252, 3436-3452 (2012)

11. Agarwal, RP, O'Regan, D, Taoudi, MA: Browder-Krasnosel'skii type fixed point theorems in Banach spaces. Fixed Point Theory Appl. (2010). doi:10.1155/2010/243716

12. Djebali, S, Sahnoun, Z: Nonlinear alternatives of Schauder and Krasnosel'skij types with applications to Hammerstein integral equations in $L^{1}$ spaces. J. Differ. Equ. 249, 2061-2075 (2010)

13. Ben Amar, A, Garcia-Falset, J: Fixed point theorems for 1-set weakly contractive and pseudocontractive operators on an unbounded domain. Port. Math. 68(2), 125-147 (2011)

14. Agarwal, RP, O'Regan, D, Taoudi, MA: Fixed point theorems for ws-compact mappings in Banach spaces. Fixed Point Theory Appl. 2010, Article ID 183596 (2010)

15. Megginson, RE: An Introduction to Banach Space Theory. Springer, Berlin (1991)

16. Agarwal, RP, Hussain, N, Taoudi, MA: Fixed point theorems in ordered Banach spaces and applications to nonlinear integral equations. Abstr. Appl. Anal. (2012). doi:10.1155/2012/245872

17. Boyd, D, Wong, JSW: On nonlinear contractions. Proc. Am. Math. Soc. 20, 458-464 (1969) 
18. Taoudi, MA: Krasnosel'skii type fixed point theorems under weak topology features. Nonlinear Anal. 72, 478-482 (2010)

19. Barroso, CS: Krasnosel'skii's fixed point theorem for weakly continuous maps. Nonlinear Anal. 55, 25-31 (2003)

20. Zeidler, E: Nonlinear Functional Analysis and Its Application I: Fixed-Point Theorems. Springer, Berlin (1986)

21. Lucchetti, R, Patrone, F: On Nemytskij's operator and its application to the lower semicontinuity of integral functionals. Indiana Univ. Math. J. 29(5), 703-735 (1980)

22. Moreira, DR, Teixeira, EVO: Weak convergence under nonlinearities. An. Acad. Bras. Ciênc. 75(1), 9-19 (2003)

doi:10.1186/1687-1812-2013-102

Cite this article as: Wang: Fixed-point theorems for the sum of two operators under $\omega$-condensing. Fixed Point Theory and Applications 2013 2013:102.

\section{Submit your manuscript to a SpringerOpen ${ }^{\circ}$} journal and benefit from:

- Convenient online submission

Rigorous peer review

- Immediate publication on acceptance

- Open access: articles freely available online

- High visibility within the field

- Retaining the copyright to your article 